\title{
Carotid dissection mimicking a new attack of cluster headache
}

\author{
Elisa Candeloro ${ }^{1 *}$, Isabella Canavero ${ }^{1}$, Maurizia Maurelli ${ }^{1}$, Anna Cavallini ${ }^{1}$, Natascia Ghiotto ${ }^{2}$, Paolo Vitali ${ }^{3}$ \\ and Giuseppe Micieli ${ }^{1}$
}

\begin{abstract}
Background: Symptomatic cluster headache $(\mathrm{CH})$ secondary to internal carotid artery dissection (ICAD) has been frequently reported, however, as far as we know, the coexistence of episodic $\mathrm{CH}$ and acute symptomatic $\mathrm{CH}$ secondary to ICAD has not.

Case report: $\mathrm{A} 39$ year-old man, affected by episodic $\mathrm{CH}$ since the age of 19, presented an atypical headache associated with his usual autonomic symptoms. After a series of negative tests, MRA eventually revealed dissection of the right distal internal carotid artery.

Discussion and conclusions: The coexistence of episodic $\mathrm{CH}$ and acute $\mathrm{CH}$ symptomatic of ICAD in our patient suggests that, at least in some cases, CH and ICAD may be different expressions of a common underlying cause: hidden vessel wall damage. When risk factors and the change - though partial - of clinical features suggest symptomatic cases, $\mathrm{CH}$ patients have to be strictly monitored over time. Given the lack of a gold standard investigation for dynamic diseases such as dissections, these patients require multimodal diagnostic investigation over time, even in cases where exams are normal at onset.
\end{abstract}

Keywords: Carotid dissection; Cluster headache; Dissection mimics

\section{Background}

It is widely recognized that carotid dissection could simulate a cluster headache $(\mathrm{CH})$ attack. In fact, many cases of symptomatic $\mathrm{CH}$ secondary to internal carotid artery dissection (ICAD) have been reported [1-5]. However, to our knowledge, the occurrence of acute symptomatic $\mathrm{CH}$ secondary to ICAD in a patient affected by episodic $\mathrm{CH}$ has never been reported. We speculate about a pathogenetic connection between the two conditions in our patient. The peculiarity of the case also offers an example of a challenging differential diagnosis in the emergency department setting.

\section{Case presentation}

On 8th March 2012, a 39 year-old man presented to the emergency department 12 hours after the onset of right

\footnotetext{
* Correspondence: elisa.candeloro@mondino.it

${ }^{1}$ Cerebrovascular Diseases and Stroke Unit, Department of Emergency Neurology, IRCCS National Institute of Neurology Foundation Casimiro Mondino, Pavia, Italy

Full list of author information is available at the end of the article
}

orbital, enduring, pressing pain. Neurological examination revealed miosis and ptosis in the right eye.

From the age of 19 the patient had suffered from episodic cluster headache, with attacks of right-orbital boring pain, lasting about 30 minutes, with conjunctival injection, rhinorrea, miosis and ptosis in the right eye. The condition was responsive to sumatriptan and verapamil. His last cluster attack had occurred in May 2011. In previous years he had undergone several neuroimaging examinations (computed tomography, magnetic resonance imaging, magnetic resonance angiography), showing no pathological findings. Past medical history also revealed a childhood post-traumatic fracture of the right petrous apex; MTHFR C677T homozygosis. The patient was practicing sport intensively, including the use of fitness equipment that implied the repetitive flexionextension of the neck.

The atypically enduring pain led us to exclude secondary forms of cluster headache. An urgent brain computed tomography (CT) with computed tomography angiography (CTA) of intra-extracranial vessels was performed in the emergency department. CTA showed

\section{实}


only a subtle asymmetry of the internal carotid lumen filling by contrast (Figure 1A, 1B) and was reported as negative for artery dissection.

Due to persisting pain and Horner's syndrome, the man was admitted to the neurology department for further investigations. On 9th March he underwent a Duplex sonography of the supra-aortic vessels and a transcranial Doppler; the findings were normal (complete filling of the ICA lumen with normal Pulse Repetition Frequency [Figure 2A] and a normal waveform [Figure 2C]). A few hours later, a brain magnetic resonance imaging (MRI) with an extra-intracranial magnetic resonance angiography (MRA) demonstrated an intramural hematoma of the right distal internal carotid (about one centimeter before the intracranial segment), without stenosis of the vessel (Figure 3A, 3B). He was immediately anticoagulated with heparin in order to prevent a stroke.

During hospitalization, the patient developed arterial hypertension, bradycardia and transient paresthesias of the right hemiface. For this reason, on the 5th day Duplex sonography was performed and disclosed a steno-occlusive distal process: the investigation disclosed the complete filling of the lumen with Pulse Repetition Frequency (Figure 2B) and a waveform with a very low amplitude, high-resistance and no diastolic flow (Figure 2D); the increased pulsatility and notable reduction in blood flow amplitude and velocity were consistent with the development of a stenosis downstream and suggested a possible worsening of carotid dissection. An extra-intracranial MRA showed apparent carotid occlusion (Figure 3C) and DWI sequences on MRI and ruled out the occurrence of a stroke. On the 10th day subtotal carotid recanalization was documented by MRA (Figure 3D). At the six-month follow up, neurological examination was normal and neuroimaging showed stable partial recanalization.

\section{Discussion}

To our knowledge, a coexistence of episodic $\mathrm{CH}$ and acute symptomatic $\mathrm{CH}$ secondary to ICAD has never been previously reported.

$\mathrm{CH}$ and ICAD could present with unilateral orbital pain in association with ipsilateral miosis and ptosis due to sympathetic dysfunction. Either in $\mathrm{CH}$ or in ICAD, rarely, it could be found also unilateral tearing, conjunctival injection and rhinorrhea due to parasympathetic dysfunction $[4,5]$.

In both $\mathrm{CH}$ and ICAD, the pericarotid plexus is involved in autonomic symptoms and trigeminal fibers in pain and parasympathetic symptoms [1,4].

According to some authors, in $\mathrm{CH}$ the simultaneity of pain and autonomic symptoms is due to a "pathophysiologic focus" in the superior pericarotid cavernous sinus plexus [6], where the fibers of the trigeminal nerve, superior cervical ganglion and sphenopalatine ganglion take connection [1-7]. Evidence for such a "peripheral" origin of symptoms comes from the observation that clinical features of symptomatic forms secondary to the involvement of these structures are almost indistinguishable from those in primary $\mathrm{CH}[8]$.

In ICAD, local signs and symptoms are thought to derive from the involvement of the adjacent structures. Autonomic symptoms could derive from the stretching and disruption of the pericarotid sympathetic nerve

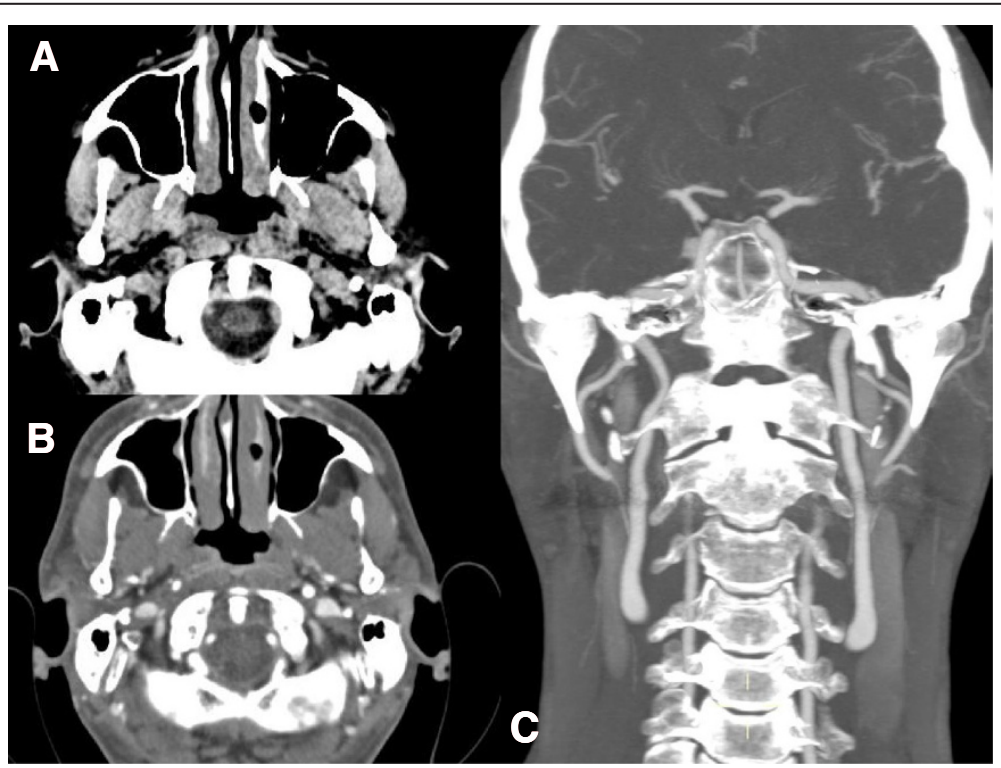

Figure 1 CT findings (performed in emergency department). A. Non-contrast axial $C T$ shows normal findings. B. Axial $C T A$ shows only a subtle asymmetry of the internal carotid lumen filling by contrast and was reported as negative for artery dissection. C. Coronal CTA shows normal findings. 


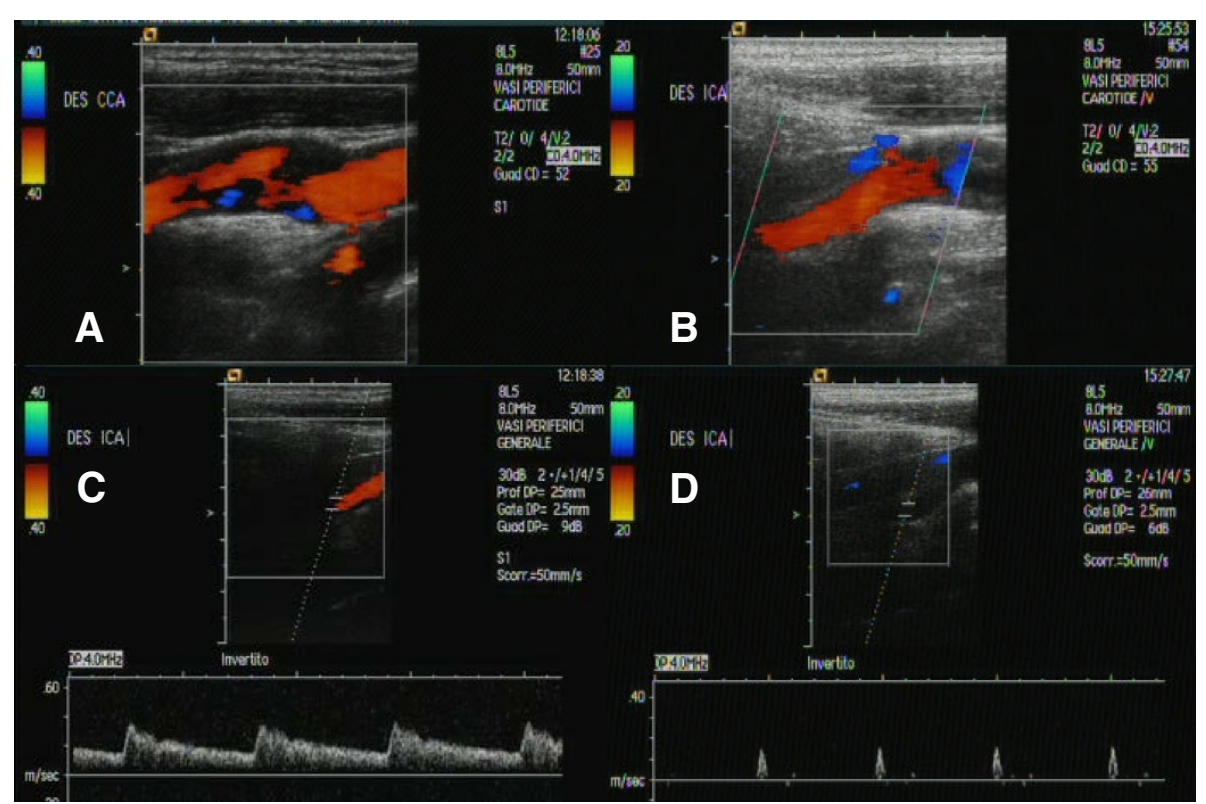

Figure 2 Duplex sonography findings. A. B-mode image performed on 9th March: complete filling of the right ICA lumen with normal PRF. B. B-mode image performed on 14th March: complete filling of the right ICA lumen with normal PRF. C. Doppler flow measurement performed on 9th March: normal waveform. D. Doppler flow measurement performed on 14th March: a waveform with a very low amplitude, high-resistance and no diastolic flow.

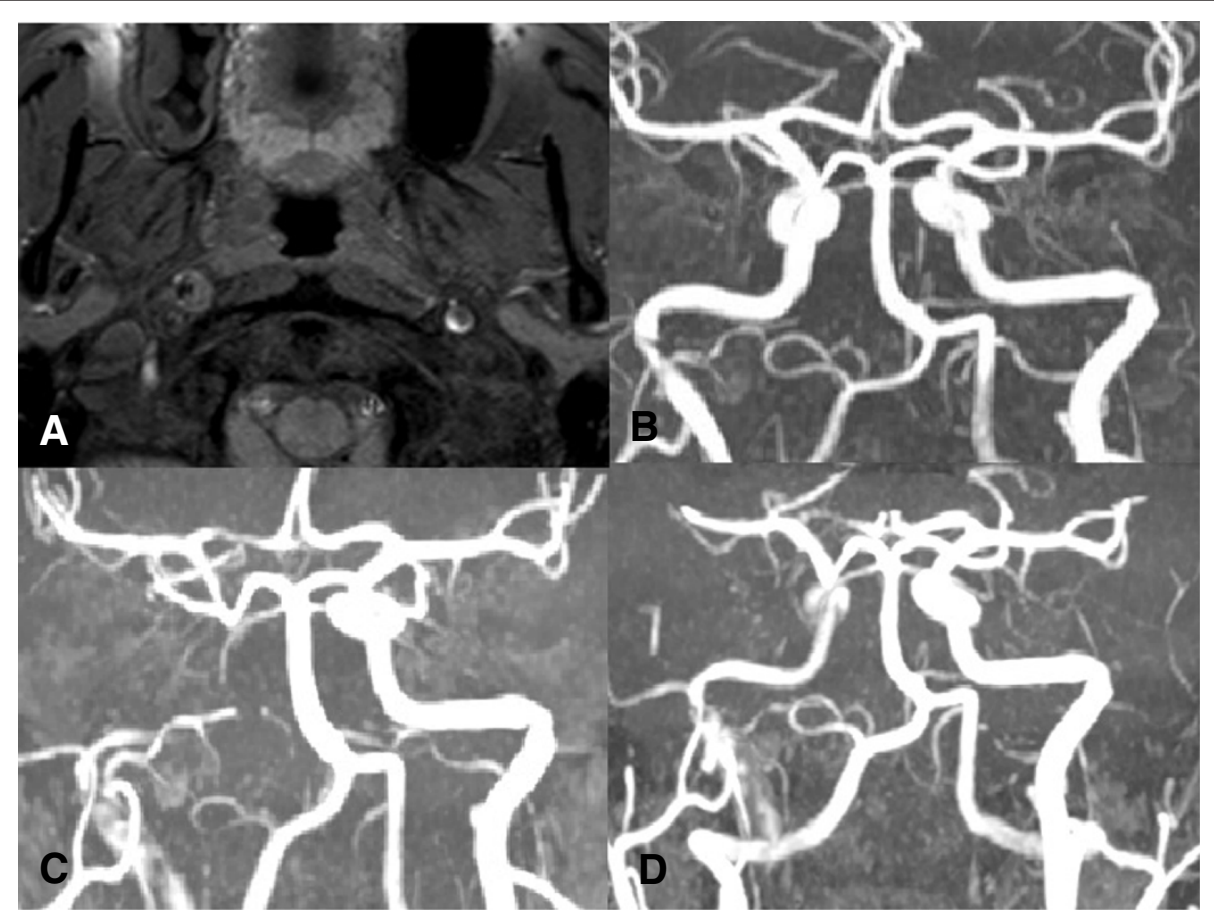

Figure 3 MR findings. A. Axial T1 fat-sat image (performed on 9th March) shows the typical semilunar image of the intramural hematoma in the right ICA. B. Time-of-flight (TOF) MRA (performed on 9th March) shows a focal "minus" image at the extra-intracranial passage in the right ICA. C. On 14th March MRA shows extension of contrastographic defect in the right ICA, due to the cranio-caudal extension of the intramural hematoma. D. On 19th March MRA shows a subtotal re-canalisation of the right ICA. 
fibers, due to the enlargement of the vessel by intramural hematoma [9]. Pain could be determined by stimulation of the trigemino-vascular system [3]. An enlarged vessel can also determine the compression of other contiguous structures such as the carotid sinus and the trigeminal nerve. In our patient, the compression of these structures resulted in "baroceptor failure syndrome", with hypertension and bradycardia, and transient parestesias in the right hemiface.

In ICAD pathogenesis, it has been hypothesized that an interaction between genetic (e.g. MTHFR C677T homozygosis) and environmental factors (e.g. craniocervical traumas, mechanical stresses during sports activity and chiropractic manipulation) may lead to the initial vessel wall damage [10].

Therefore, in our case, the presence of several of these risk factors (the post-traumatic petrous apex fracture, MTHFR C677T homozygosis and repeated mechanical stress during sports activity), the coexistence of $\mathrm{CH}$ and ICAD with overlapping clinical features and the inferable involvement of the same afore-mentioned anatomical structures, suggest that the two diseases could share a common "pathophysiologic focus": probably hidden vessel wall damage.

A clinical differential diagnosis between $\mathrm{CH}$ and ICAD is influenced by the similarity of the symptoms, even more so if the two conditions co-exist. In ICAD pain is often associated with ipsilateral Horner syndrome, while other autonomic symptoms are unusual. However, in literature it has been described a case of ICAD whose $\mathrm{CH}$-like symptoms were Horner syndrome, tearing and rhinorrhea [4]. The time course of pain was the only feature to be distinctive from $\mathrm{CH}$, as in our patient.

Clinical observation and monitoring are crucial in order to note every new, atypical feature of symptoms such as, in our case, enduring pain.

However, anamnestic and clinical data could be unable to support the differential diagnosis, as reported by Godeiro-Junior et al. [5]: in their report ICAD presented with $\mathrm{CH}$-like pain, Horner syndrome and other autonomic symptoms. For these reasons, further investigations are mandatory.

Nevertheless, even instrumental differential diagnosis is hindered by some factors: the heterogeneous anatomopathological features of vessel wall injury, the high dynamicity of dissections, and the intrinsic limits of the available instrumental techniques (especially in emergency departments).

Some Authors have proposed that spontaneous cervical artery dissections affect primarily the outer arterial layers, taking origin from a degenerative process at the medial-adventitial border. This may lead to the formation of a neoangiogenetic network and, subsequently, intramural hematomas [11]. The direction of expansion of the intramural hematoma determines the successive steps: if it is towards the lumen, stenosis or occlusion of the vessel could occur, with, though not necessarily, the formation of a double-lumen and an intimal flap; if it is towards the surrounding tissues, it could produce dilatation of the vessel and cause compressive local signs. Intimal flap, double lumen, pseudoaneurysm are considered pathognomonic of arterial dissection, but not invariably found. Their absence does not exclude the diagnosis. Further, it is difficult to predict if dissection will evolve into arterial steno-occlusion or recanalization; the timing of these changes could be even harder to predict [12]. It follows that the assumption that there is a correspondence between the time of the onset of dissection and the time of the onset of symptoms and signs may not be true in all cases [13], as the incidental finding of asymptomatic cases demonstrates.

The high anatomical heterogeneity and the unpredictable natural course of ICAD hinder instrumental diagnoses, especially in the absence of a gold standard test [13]. The available techniques are able to evaluate different aspects of the disease. CTA investigates mainly the lumen; it has a good sensitivity for stenosis, intimal flap and pseudoaneurysm but poor sensitivity for isolated intramural hematoma [14]. Owing to the mostly distal location of ICAD, often only indirect signs are detectable with Duplex sonography. This technique assesses a reduction of blood flow with good sensitivity only with stenosis $>50 \%$ downstream; ultrasound investigation has poor sensitivity in non-stroke patients with isolated Horner syndrome [15]. B-mode images visualize the arterial wall and the surrounding tissue: they may identify the double lumen but with poor sensitivity and only when located in the proximal extracranial carotid segment [16]. MRA evaluates the vessel wall and is able to detect intramural hematoma despite vessel occlusion: axial MRA T1-weighted imaging with fat suppression allows an optimal discrimination between intramural hematoma and perivascular tissue [17].

At the onset of symptoms in our patient the only sign of artery dissection was an isolated intramural hematoma, as disclosed by MRA; CTA was reported as normal given the absence of stenosis/intimal flap/ pseudoaneurysm and Duplex sonography was normal due to the distal non-stenosing localization of the injury. Over the following days, ultrasounds showed abnormal findings due to the development of a significant stenosis that was confirmed by MRA.

\section{Conclusions}

Our case suggests that, in some cases, episodic $\mathrm{CH}$ and symptomatic $\mathrm{CH}$ in ICAD could share a common etiopathogenetic mechanism. This being, to our knowledge, the first case of association between $\mathrm{CH}$ and 
ICAD, and considering the available current literature $[8,13]$, we believe that this hypothesis deserves attention and requires further studies to be confirmed.

From this report we could infer that patients with typical $\mathrm{CH}$ attacks [18], in the presence of risk factors for vessel wall damage, should undergo a careful clinical-instrumental follow up, paying particular attention to the development of atypical clinical features, such as pain.

This case highlights the need to combine different investigations and repeat them over time in $\mathrm{CH}$ patients who develop new atypical symptoms suggestive of ICAD. In fact, a negative initial investigation does not rule out the diagnosis of dissection.

\section{Consent}

Written informed consent was obtained from the patient for publication of this Case report and the accompanying images. A copy of the written consent is available for review by the Editor-in-Chief of this journal.

\section{Abbreviations}

CH: Cluster headache; ICA: Internal carotid artery; ICAD: Internal carotid artery dissection; MRI: Magnetic resonance imaging; MRA: Magnetic resonance angiography; CT: Computed tomography; CTA: Computed tomography angiography; PRF: Pulse repetition frequency.

\section{Competing interests}

The authors declare that they have no competing interests.

\section{Authors' contributions}

CE conceived of the study, participated in clinical management of the patient, reviewed the literature on the item and drafted the manuscript. Cl participated in the design of the paper, in reviewing the literature and drafting the manuscript. MM had the first approach with the patient at the $E R$, made the correct diagnosis and participated in his clinical management. CA has contributed in the clinical management of the patient and revised the manuscript. GN performed the neurosonological investigation and reviewed the literature concerning the technique. VP performed the neuroradiological investigation and reviewed the literature concerning the technique. MG has contributed in the clinical management of the patient and revised the manuscript. All authors read and approved the final manuscript.

\section{Author details}

${ }^{1}$ Cerebrovascular Diseases and Stroke Unit, Department of Emergency Neurology, IRCCS National Institute of Neurology Foundation Casimiro Mondino, Pavia, Italy. ${ }^{2}$ Neurosonology Unit, Department of Neuropathophysiology, IRCCS National Institute of Neurology Foundation Casimiro Mondino, Pavia, Italy. ${ }^{3}$ Department of Neuroradiology, IRCCS National Institute of Neurology Foundation Casimiro Mondino, Pavia, Italy.

\section{Received: 12 July 2013 Accepted: 19 September 2013}

Published: 8 October 2013

\section{References}

1. Hannerz J, Arnardottir S, Bro Skejø HP, Lilja JA, Ericson K (2005) Peripheral postganglionic sympathicoplegia mimicking cluster headache attacks. Headache 45(1):84-86

2. Frigerio S, Bühler R, Hess CW, Sturzenegger M (2003) Symptomatic cluster headache in internal carotid artery dissection--consider anhidrosis. Headache 43(8):896-900

3. Biousse V, D'Anglejan-Chatillon J, Massiou H, Bousser MG (1994) Head pain in non-traumatic carotid artery dissection: a series of 65 patients. Cephalalgia 14(1):33-36
4. Tsivgoulis G, Mantatzis M, Vadikolias K, Heliopoulos I, Charalampopoulos K, Mitsoglou A, Georgiadisa GS, Giannopoulos S, Piperidou C (2013) Internal carotid artery dissection presenting as new-onset cluster headache. Neurol Sci 34(7):1251-1252

5. Godeiro-Junior C, Kuster GW, Felicio AC, Porto PP Jr, Pieri A, Coelho FM (2008) Internal carotid artery dissection presenting as cluster headache. Arq Neuropsiquiatr 66(3):763-764

6. Moskowitz MA (1988) Cluster headache: evidence for a pathophysiologic focus in the superior pericarotid cavernous sinus plexus. Headache 28(9):584-586

7. Gentile S, Fontanella M, Giudice RL, Rainero I, Rubino E, Pinessi L (2006) Resolution of cluster headache after closure of an anterior communicating artery aneurysm: the role of pericarotid sympathetic fibres. Clin Neurol Neurosurg 108(2):195-198

8. Leone M, Bussone G (2009) Pathophysiology of trigeminal autonomic cephalalgias. Lancet Neurol 8(8):755-764

9. Sturzenegger M, Huber $P$ (1993) Cranial nerve palsies in spontaneous carotid artery dissection. J Neurol Neurosurg Psychiatry 56(11):1191-1199

10. Debette S, Grond-Ginsbach C, Bodenant M, Kloss M, Engelter S, Metso T, Pezzini A, Brandt T, Caso V, Touzé E, Metso A, Canaple S, Abboud S, Giacalone G, Lyrer P, Del Zotto E, Giroud M, Samson Y, Dallongeville J, Tatlisumak T, Leys D, Martin JJ, Cervical Artery Dissection Ischemic Stroke Patients (CADISP) Group (2011) Differential features of carotid and vertebral artery dissections: the CADISP study. Neurology 77(12):1174-81

11. Völker W, Dittrich R, Grewe S, Nassenstein I, Csiba L, Herczeg L, Borsay BA, Robenek H, Kuhlenbäumer G, Ringelstein EB (2011) The outer arterial wall layers are primarily affected in spontaneous cervical artery dissection. Neurology Apr 76:1463-1471

12. Sengelhoff C, Nebelsieck J, Nassenstein I, Maintz D, Nabavi DG, Kuhlenbaeumer G, Ringelstein EB, Dittrich R (2008) Neurosonographical follow-up in patients with spontaneous cervical artery dissection. Neurol Res 30(7):687-689

13. Provenzale JM, Sarikaya B (2009) Comparison of test performance characteristics of MRI, MR angiography, and CT angiography in the diagnosis of carotid and vertebral artery dissection: a review of the medical literature. AJR Am J Roentgenol 193(4):1167-1174

14. Goyal MS, Derdeyn CP (2009) The diagnosis and management of supraaortic arterial dissections. Curr Opin Neurol 22(1):80-89

15. Arnold M, Baumgartner RW, Stapf C, Nedeltchev K, Buffon F, Benninger D, Georgiadis D, Sturzenegger M, Mattle HP, Bousser MG (2008) Ultrasound diagnosis of spontaneous carotid dissection with isolated Horner syndrome. Stroke 39(1):82-86

16. Benninger DH, Baumgartner RW (2006) Ultrasound diagnosis of cervical artery dissection. Front Neurol Neurosci 21:70-84

17. Ozdoba C, Sturzenegger M, Schroth G (1996) Internal carotid artery dissection: MR imaging features and clinical-radiologic correlation. Radiology 199(1):191-198

18. Headache Classification Committee of the International Headache Society (IHS) (2013) The International Classification of Headache Disorders, 3rd edition (beta version). Cephalalgia 33(9):629-808

doi:10.1186/1129-2377-14-84

Cite this article as: Candeloro et al:: Carotid dissection mimicking a new attack of cluster headache. The Journal of Headache and Pain 2013 14:84.

\section{Submit your manuscript to a SpringerOpen ${ }^{\odot}$ journal and benefit from:}

- Convenient online submission

- Rigorous peer review

- Immediate publication on acceptance

- Open access: articles freely available online

- High visibility within the field

- Retaining the copyright to your article

Submit your next manuscript at $\gg$ springeropen.com 